\title{
Patterns and Predictors of Early Mortality in Incident Hemodialysis Patients: New Insights
}

\author{
Lilia R. Lukowsky ${ }^{a}$ b Leeka Kheifets ${ }^{b}$ Onyebuchi A. Arah ${ }^{b, e}$ \\ Allen R. Nissenson $^{c, d}$ Kamyar Kalantar-Zadeh ${ }^{\mathrm{a}-\mathrm{c}}$ \\ ${ }^{a}$ Harold Simmons Center for Chronic Disease Research and Epidemiology, Los Angeles Biomedical Research \\ Institute at Harbor-UCLA Medical Center, Torrance, Calif., ' Department of Epidemiology, UCLA Fielding School of \\ Public Health, and 'David Geffen School of Medicine at UCLA, Los Angeles, Calif., and ' DaVita, Inc., \\ El Segundo, Calif., USA; 'Department of Public Health, Academic Medical Center, University of Amsterdam, \\ Amsterdam, The Netherlands
}

\section{Key Words}

Incident hemodialysis patients • Mortality predictor •

Population attributable fraction

\begin{abstract}
Background: Incident hemodialysis patients have the highest mortality in the first several months after starting dialysis treatments. We hypothesized that the patterns and risk factors associated with this early mortality differ from those in later dialysis therapy periods. Methods: We examined mortality patterns and predictors during the first several months of hemodialysis treatment in 18,707 incident patients since the first week of hemodialysis therapy and estimated the population attributable fractions for selected time periods in the first 24 months. Results: The 18,707 incident hemodialysis patients were $45 \%$ women and $54 \%$ diabetics. The standardized mortality ratios (95\% confidence interval) in the 1st to 3rd month of hemodialysis therapy were 1.81 (1.74$1.88), 1.79(1.72-1.86)$, and 1.34 (1.27-1.40), respectively. The standardized mortality ratio reached prevalent mortality only by the 7th month. No survival advantage for African Americans existed in the first 6 months. Patients with low albumin $<3.5 \mathrm{~g} / \mathrm{dl}$ had the highest proportion of infection-
\end{abstract}

related deaths while patients with higher albumin levels had higher cardiovascular deaths including $76 \%$ of deaths during the first 3 months. Use of catheter as vascular access and hypoalbuminemia $<3.5 \mathrm{~g} / \mathrm{dl}$ explained $34 \%(17-54 \%)$ and $33 \%$ $(19-45 \%)$ of all deaths in the first 90 days, respectively. Conclusions: Incident hemodialysis patients have the highest mortality during the first 6 months including 80\% higher death risk in the first 2 months. The presence of a central venous catheter and hypoalbuminemia $<3.5 \mathrm{~g} / \mathrm{dl}$ each explain one third of all deaths in the first 90 days.

Copyright $\odot 2012$ S. Karger AG, Basel

\section{Introduction}

The number of patients with end-stage renal disease (ESRD) in the United States has increased from about 60,000 in 1980 to over half a million in 2008 [1]. It is projected that this number would surpass 800,000 by 2020

This paper serves as the main component of the doctoral thesis of Ms. Lilia R. Lukowsky for a PhD degree in Epidemiology at UCLA School of Public Health. Other coauthors are the members of the thesis committee.

\section{KARGER}

Fax +41613061234

E-Mail karger@karger.ch

www.karger.com (c) 2012 S. Karger AG, Basel

$0250-8095 / 12 / 0356-0548 \$ 38.00 / 0$

Accessible online at:

www.karger.com/ajn
Kamyar Kalantar-Zadeh, MD, PhD, MPH

Harold Simmons Center for Chronic Disease

Division of Nephrology and Hypertension, Harbor-UCLA Medical Center

1124 West Carson Street, C1-Annex, Torrance, CA 90509-2910 (USA)

Tel. +1 310222 3891, E-Mail kamkal@ucla.edu 
[2]. These patients would not have survived without kidney transplantation or dialysis therapy, which currently comprises mostly of hemodialysis treatment in this country. However, dialysis patient mortality is unacceptably high, currently approximately $20 \%$ per year in the United States. Mortality appears to be even higher during the first year of dialysis therapy, especially in the first few months [2], while factors contributing to early death are widely unknown.

The most common cause of death in dialysis patients is cardiovascular followed by infectious disease. In a recent study that examined the early mortality among incident hemodialysis patients during the first 120 days versus the subsequent 121 to 365 days [3], cardiovascular causes were still the most common for the entire first year. Even though previous studies identified several important factors associated with elevated mortality among incident hemodialysis patients, few have addressed the risk factor patterns and their changes over time during the first few months of dialysis therapy. It is crucial to assess whether those risks remain constant or whether the risk patterns are altered over time, so that focused interventions can be used at different periods of time. Comorbidities including severity of kidney disease at the time of dialysis initiation could play an important role in survival of dialysis patients [3]. Several recent observational studies [4-6] and at least one randomized trial [7] have indicated that a higher glomerular filtration rate at the time of dialysis initiation was associated with elevated mortality risk in dialysis patients, although this may be due to the fact that sicker and older patients start dialysis therapy earlier [8]. Use of central venous catheters (CVC), which exits in up to $82 \%$ patients at the start of hemodialysis therapy [9-12], has been implicated as an important mortality predictor in several studies $[9,12]$.

We hypothesized that mortality rate is substantially higher during the first few months of dialysis initiation and that the risk factors associated with this high mortality are different from those in later periods of dialysis therapy. We examined the associations between the mortality and the putative risk factors during different time periods within the first 24 months after the start of hemodialysis therapy among a large group of incident hemodialysis patients who had started treatment at one of the DaVita dialysis facilities in the United States between July 2001 and June 2006 and whose clinical outcomes were followed during the 5 -year period.

Early Death in Hemodialysis

\section{Methods}

Sources of Data, Study Population and Follow-Up

$\mathrm{DaV}$ ita is one of the largest dialysis providers in United States. For this study we examined the cohort of all incident hemodialysis patients who started the first week of hemodialysis treatment from July 1, 2001, to June 30, 2006, at one of the DaVita dialysis centers. Patients who used peritoneal dialysis modality at any given time were excluded. Information about the date when the patient entered the DaVita cohort, dialysis treatment modality, date when the first hemodialysis treatment started, demographic characteristics, comorbidities, and laboratory and other clinical measures were collected at the time of the start of enrollment in DaVita. Using unique identifiers, data from DaVita and the United States Renal Data System (USRDS) databases were crosslinked to corroborate the information about dates of the events including death and transplantation, and comorbidities at the start of dialysis therapy, dialysis modality and laboratory data prior to the dialysis treatment were verified as well [13]. To examine the patterns of survival in the first 2 years of hemodialysis treatment, the cohort time was divided into a priori selected smaller groups, i.e., $<3$ months, 3 to $<6$ months, 6 to $<12$ months, and 12 to 24 months. Patients were followed for up to 5 years (1,830 days) or until death, kidney transplantation, or the end of the follow-up. Person-time was obtained by calculating the difference between the dates when the first hemodialysis treatment started at one of the DaVita clinics and the end of follow-up or other censoring events.

\section{Laboratory Parameters}

All blood samples were drawn using standardized procedure and transported to the Central DaVita Laboratory located in Deland, Fla. usually within $24 \mathrm{~h}$. Blood or serum levels of albumin, creatinine, phosphorus, calcium, bicarbonate, total iron-binding capacity (TIBC), iron (transferrin) saturation ratio (ISAT or TSAT, i.e., iron divided by TIBC), white blood cell count (WBC), and lymphocyte counts were usually measured monthly. Serum ferritin was measured at least quarterly. Hemoglobin was measured weekly to biweekly. Estimate of prescribed dialysis treatment dose, known as Kt/V (single pool) [14], and protein intake, known as normalized protein catabolic rate (nPCR) [15], were obtained using urea dynamic equations. All measurements were averaged over the first calendar quarter (up to 13 weeks) to calculate one single value for each laboratory parameter per each patient. Since most of these laboratory measurements (albumin, creatinine, phosphorus, calcium, bicarbonate, TIBC, WBC, lymphocyte, ferritin, and nPCR) and body mass index (BMI) may reflect the nutritional and/or inflammatory status of dialysis patients, they are referred to as the 'malnutrition-inflammation cachexia syndrome' (MICS) throughout this study [16].

In order to calculate summary estimates of the exposure variability as putative risk of death in a clinically relevant and commensurate format, we rescaled some laboratory measures by defining biologically and clinically meaningful increments including $0.2 \mathrm{~g} / \mathrm{dl}$ of albumin, $0.2 \mathrm{~g} / \mathrm{kg} / \mathrm{day}$ of $\mathrm{nPCR}, 2 \mathrm{mEq} / \mathrm{l}$ of bicarbonate, 2 index points of BMI, $10 \%$ of lymphocyte percentage, $100 \mathrm{pg} / \mathrm{ml}$ of parathyroid hormone, $10 \%$ of ISAT, $50 \mathrm{mg} / \mathrm{dl}$ of TIBC, $500 \mathrm{ng} / \mathrm{ml}$ of ferritin, and $5 \times 10^{3} / \mathrm{HPF}$ of WBC. For other laboratory values, one conventional unit was used such as $1 \mathrm{mg} /$ $\mathrm{dl}$ increase in serum calcium or phosphorus concentrations. Age 
was also examined as decades of increments. Additionally we also examined the associations between the mortality and clinically relevant dichotomies for selected laboratory variables including serum albumin (comparing mortality for patients with $<3.5 \mathrm{vs.}$ $\geq 3.5 \mathrm{~g} / \mathrm{dl}$ ), hemoglobin ( $\geq 10 \mathrm{vs.}<10 \mathrm{~g} / \mathrm{dl}$ ), and nPCR ( $\geq 1$ vs. $<1 \mathrm{~g} / \mathrm{kg} /$ day)

\section{Statistical Analyses}

Descriptive analyses were conducted to examine the population characteristics across the a priori selected survival periods of $<3$ months, 3 to $<6$ months, 6 to $<12$ months and 12 to $<24$ months. Estimated hazard functions were examined by the life table methods. Five-year unadjusted and adjusted survival curves using Kaplan-Meier (KM) estimation were produced for the entire population as well as for the important demographic characteristics and comorbidities. Survival curves were adjusted for four main demographic features including age, gender, presence or absence of diabetes mellitus upon dialysis therapy and race, i.e., African Americans vs. others. We also calculated monthly standardized mortality ratios (SMRs) for each of the first 24 months of dialysis treatment for the incident hemodialysis patients under study. The rates were standardized to age, gender, diabetes status, and race using the cohort of all other patients who started hemodialysis treatment in a DaVita clinic within 90 days from dialysis initiation. Multivariate logistic regression models were fit in order to estimate the SMR for each month [17]. Cox proportional hazard models were used to calculate hazard ratios of death at different time periods during the 24 months and for the 5 -year survival for the patient characteristics including demographics, comorbidities and laboratory values. For each selected period, deaths before and after the period were censored and the person-time was limited to the given period.

Two levels of multivariate adjustments were used in most survival analyses: (1) Case-mix-and dialysis treatment-adjusted models, which included adjustment for age, gender, four mutually exclusive race/ethnicity categories (African Americans, Hispanics, non-Hispanic whites, and others), primary insurance (Medicare, Medicaid, and other), marital status (married, single, divorced, widowed), dialysis vascular access, i.e., CVC, arteriovenous fistula, or arteriovenous graft; dialysis dose (single pool Kt/V), diabetes mellitus as well as 11 additional comorbid conditions including atherosclerosis, congestive heart failure (CHF), other cardiac conditions, cerebrovascular disease, peripheral vascular disease, chronic obstructive pulmonary disease, cancer, hypertension, inability to ambulate, and smoking status; and entry calendar quarter for secular trend. (2) Fully adjusted models, which included adjustment for all of the above, as well as 10 above-mentioned laboratory surrogates of the MICS and BMI. Some of the adjustors were also examined as independent predictors of death risk.

The population attributable fractions (PAF) [18] were calculated for the relevant comorbidities and the CVC vascular access to assess the percent of deaths that could be attributable to those factors, and therefore, hypothetically preventable proportion by eliminating or reducing them. The $95 \%$ confidence interval for each PAF was calculated by the substitution method [19]. All the analyses were conducted by using SAS 9.2 software (SAS Inc., N.C., USA).

\section{Results}

From July 1, 2001 to June 30, 2006 a total of 82,566 incident (new) dialysis patients started chronic dialysis treatment in a DaVita dialysis clinic within the first 90 days of therapy initiation. After excluding patients younger than 18 years $(n=637)$, incident peritoneal dialysis patients or those who switched modality at any given time ( $\mathrm{n}=4,763)$, hemodialysis patients with missing person-time $(n=6)$ and hemodialysis patients who initiated the first week of dialysis therapy outside of a DaVita clinic ( $\mathrm{n}=58,453$, which served as the 'reference' population to calculate SMRs, see above and below), a total of 18,707 incident hemodialysis patients who had never switched modality remained in the cohort. These patients had received at least one treatment of the first therapy week in a DaVita clinic and had remained in DaVita throughout the entire first 90 days or until death or transplantation.

During the first 24 months, 6,666 patients died (36\% or 30 deaths per 100 person-years) and 1,399 received a kidney transplant. Online supplementary table $1 \mathrm{~S}$ (see www.karger.com/doi/10.1159/000338673 for all online suppl. material) compares the baseline demographic, clinical and laboratory features of the entire $18,707 \mathrm{pa}-$ tients with the reference cohort of 58,453 patients. The overall probability of survival of these incident hemodialysis patients for 3 and 6 months, and 1, 2 and 5 years were 0.90 (0.89-0.91), 0.80 (0.79-0.82), 0.72 (0.71-0.73), $0.58(0.57-0.59)$, and $0.26(0.25-0.27)$, respectively. Figure 1 shows the monthly SMRs over the first 24 months after dialysis therapy initiation in 18,707 incident hemodialysis patients under study using the cohort of the other 58,453 incident hemodialysis patients (see above) as the reference population. The highest mortality occurred during months 1 and 2, and mortality rates decreased over a period of 7 months. The calculated SMR (and 95\% confidence interval) for the 1st to 4 th month of dialysis therapy were 1.81 (1.74-1.88), 1.79 (1.72-1.86), 1.34 (1.271.40 ) and 1.35 (1.28-1.41), respectively (see fig. 1).

Examining the distribution of 6,666 deaths in the first 24 months, crude mortality rates during the 4 a priori selected periods of $<3,3$ to $<6,6$ to $<12$, and 12 to $<24$ months were 1,994 (30\% of all deaths or 47 deaths per 100 person-years), 1,271 (19\% or 35 per 100 personyears), 1,550 ( $23 \%$ or 25 per 100 person-years), and 1,851 (28\% or 22 per 100 person-years), respectively. Table 1 shows the characteristics for the deceased patients across the 4 a priori selected mortality periods of $<3,3$ to $<6$, 6 to $<12$, and 12 to $<24$ months compared to $\geq 24$-month 
Fig. 1. Monthly standardized mortality ratios (SMRs) during the first 2 years for the incident dialysis patients $(\mathrm{n}=18,707)$. SMRs are standardized by age, gender, diabetes and race using the reference cohort of 57,456 hemodialysis patients who started DaVita cohort within 30 days after starting dialysis treatment (see text).

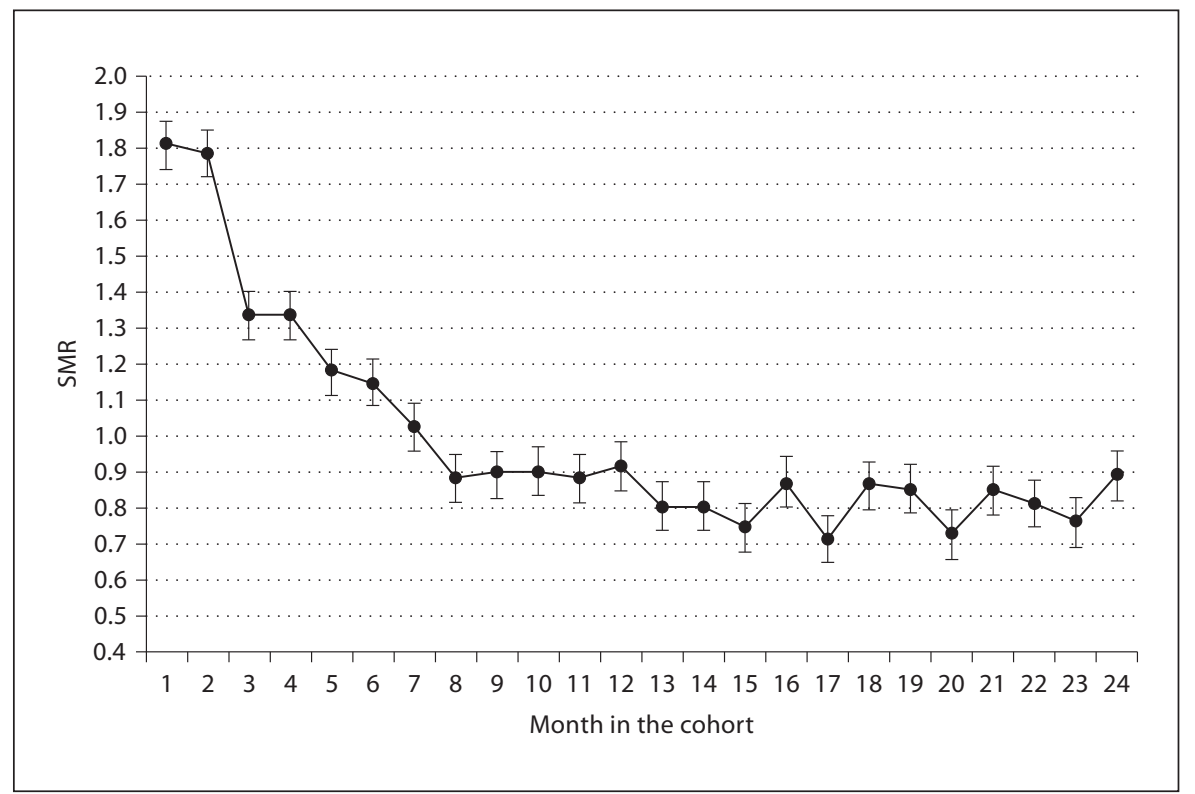

survivors. Early death was associated with more advanced age, higher proportion of CVC, and higher prevalence of cardiovascular diseases. Those who died during the first 3 months had 1.9 comorbidities per person compared to 1.1 among those who survived $\geq 2$ years. Figure 2 shows the common kidney disease etiologies, i.e., ESRD diagnoses, among patients who died during each of the a priori selected periods of the first 24 months. Diabetes and hypertension were the 2 most common ESRD diagnoses; however, hypertension was more frequent among those with earlier deaths, whereas diabetes was more frequent among those who survived the earlier months. Figure 3 shows the causes of death during the first 24 months. Nearly similar death cause distribution was noticed in the first 90 days indicating that half of all deaths were marked as cardiovascular. Mortality due to withdrawal from dialysis was the lowest during the first 90 days and rose over time from 2 to $8 \%$ in the second year. Table 2 shows case-mix-adjusted death hazard ratios during the 4 survival periods. The known survival advantage of African American race was noticeable only after 6 months of therapy but not prior to that. Diabetes mellitus and lower nPCR were paradoxically associated with greater survival at early time periods but they were predictors of higher mortality during later periods.

In addition to all-cause mortality, we also examined the associations of cause-specific mortality, i.e., cardiovascular and infection-related deaths, with race (non-
Hispanic whites, African Americans and Hispanics), history of CHF, CVC vascular access and hypoalbuminemia as presented in table 3. Compared to whites, both African Americans and Hispanics showed slightly better survival in terms of cardiovascular and infection-related mortality, although the associations were uncertain initially. Of note, patients with CHF showed elevated risk of death independently of the cause of death. Moreover the associations were higher for both cardiovascular (1.68 [1.411.99] during the first quarter) and infection-related deaths (1.56 [1.14-2.14] during the first quarter) comparing to all-cause mortality (1.31 [1.11-1.54]). Use of CVC access was associated with the deaths from infections much stronger (3.32 [2.14-5.16] during the first quarter) comparing to cardiovascular deaths. Similarly, low serum albumin levels were associated with infection-related mortality much stronger $(4.92$ [3.45-7.00] in the first 3 months) than with cardiovascular mortality during the same time period.

Online supplementary table $2 \mathrm{~S}$ shows selected PAF including for vascular access and comorbidities. CVC as the dialysis access could explain $34 \%$ of all deaths in the first 3 months of dialysis therapy. A low serum albumin $<3.5$ $\mathrm{g} / \mathrm{dl}$ had a similar death contribution, i.e., $33 \%$ of deaths in the first 90 days could have been prevented if albumin levels were above $3.5 \mathrm{~g} / \mathrm{dl}$. CHF and serum level of hemoglobin under $10 \mathrm{~g} / \mathrm{ml}$ each contributed to 10 and $9 \%$ of all deaths during the first 90 days. 
Table 1. Comparing patient characteristics across selected morality periods of the first 24 months in 18,707 incident hemodialysis patients who started dialysis therapy during July 1, 2001-June 30, 2006 in a DaVita clinic

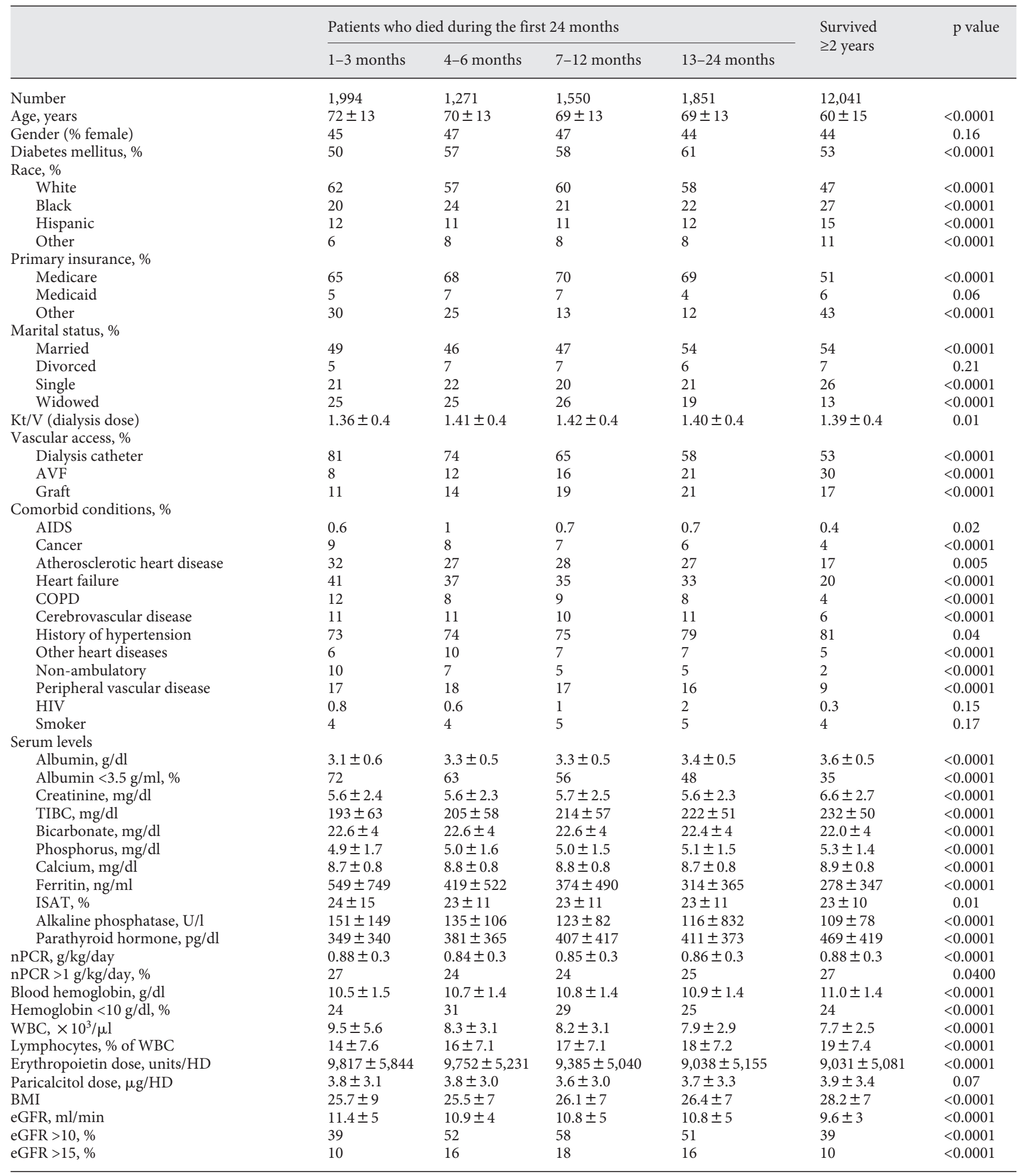

COPD = Chronic obstructive pulmonary disease; eGFR = estimated glomerular filtration rate. 


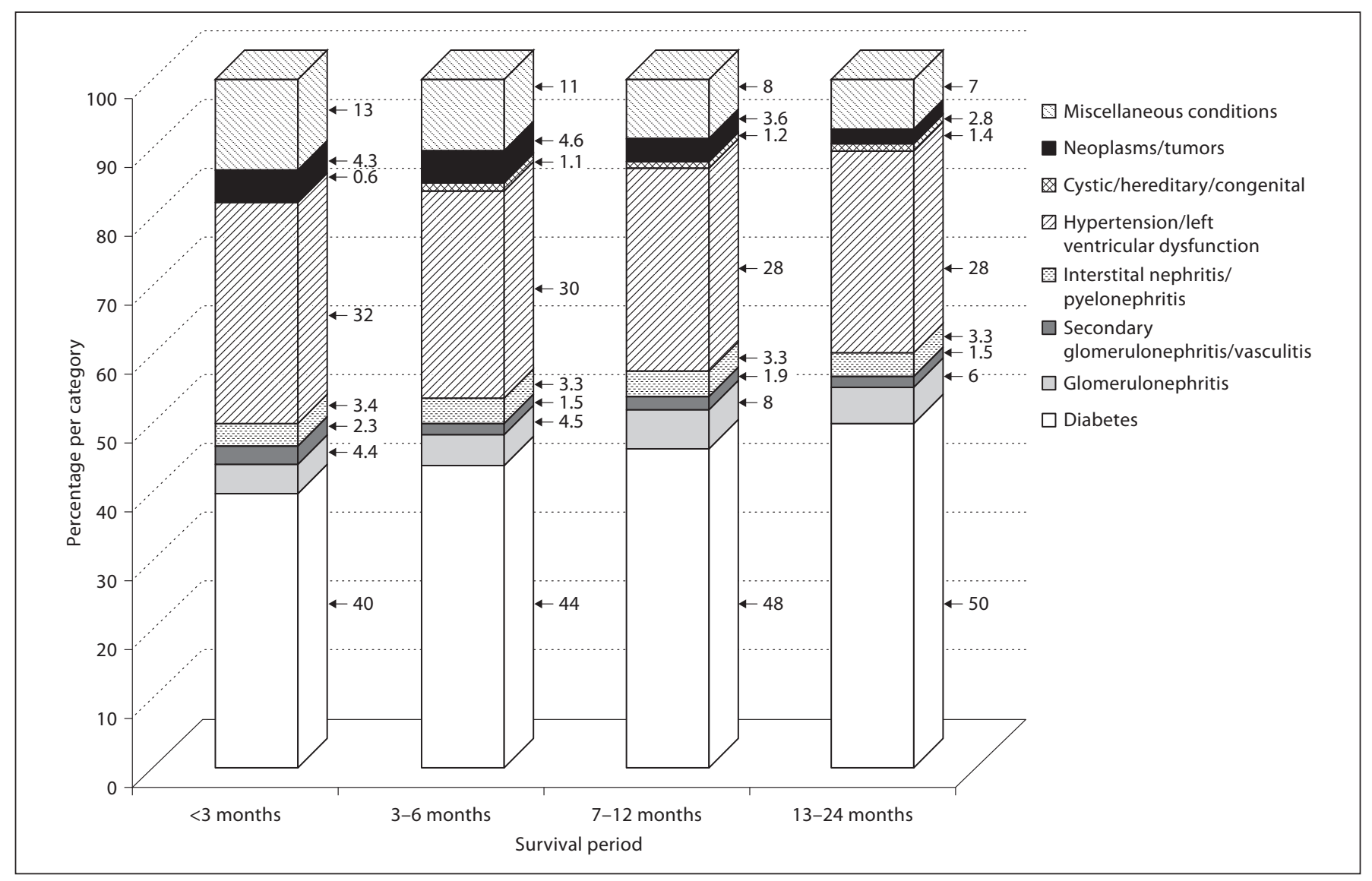

Fig. 2. Primary causes of ESRD for incident hemodialysis patients who died within 24 months after dialysis initiation from the 5-year DaVita cohort July 2001-June $2006(\mathrm{n}=6,507)$ compared to survivors (n total = 17,871).

\section{Discussion}

Examining the mortality patterns during the first 24 months of a contemporary (2001-2006) and nationally representative cohort of 18,707 incident hemodialysis patients who received treatment from the first week of hemodialysis therapy in a DaVita dialysis clinic, we found that mortality was exceptionally high in the first 6 months, especially during months 1 and 2. SMRs were 1.81 and 1.79 , respectively, compared to all incident hemodialysis patients of the same cohort period. We also assessed the risk of death during different time periods to identify modifiable risk factors and their contributions to death over time and found that use of CVC as the vascular access and hypoalbuminemia $<3.5 \mathrm{mg} / \mathrm{dl}$ could explain 34 and $33 \%$ of all deaths in the first 90 days, respectively. These novel findings may have important clinical and public health implications, since they may offer im- petus for designing interventions and trials to reduce early death among incident dialysis patients.

We found that both all-cause and cardiovascular mortality rates were the highest during the first 2 months of dialysis therapy, which is consistent with several previous reports $[3,12]$.

Among demographic predictors of death, older age, white race, Medicaid coverage, and being single or widowed were also associated with higher death risk in the first 24 months.

Worse survival of non-Hispanic white dialysis patients is consistent with previous reports $[3,20]$. Even though it remains unclear as to why African Americans have lower dialysis mortality risk, in our study the survival advantage of African Americans or other minorities was almost nonexistent in the first 6 months. Of note, the racial distribution of patients who died within the first 3 months consisted of $62 \%$ non-Hispanic whites, $20 \%$ 


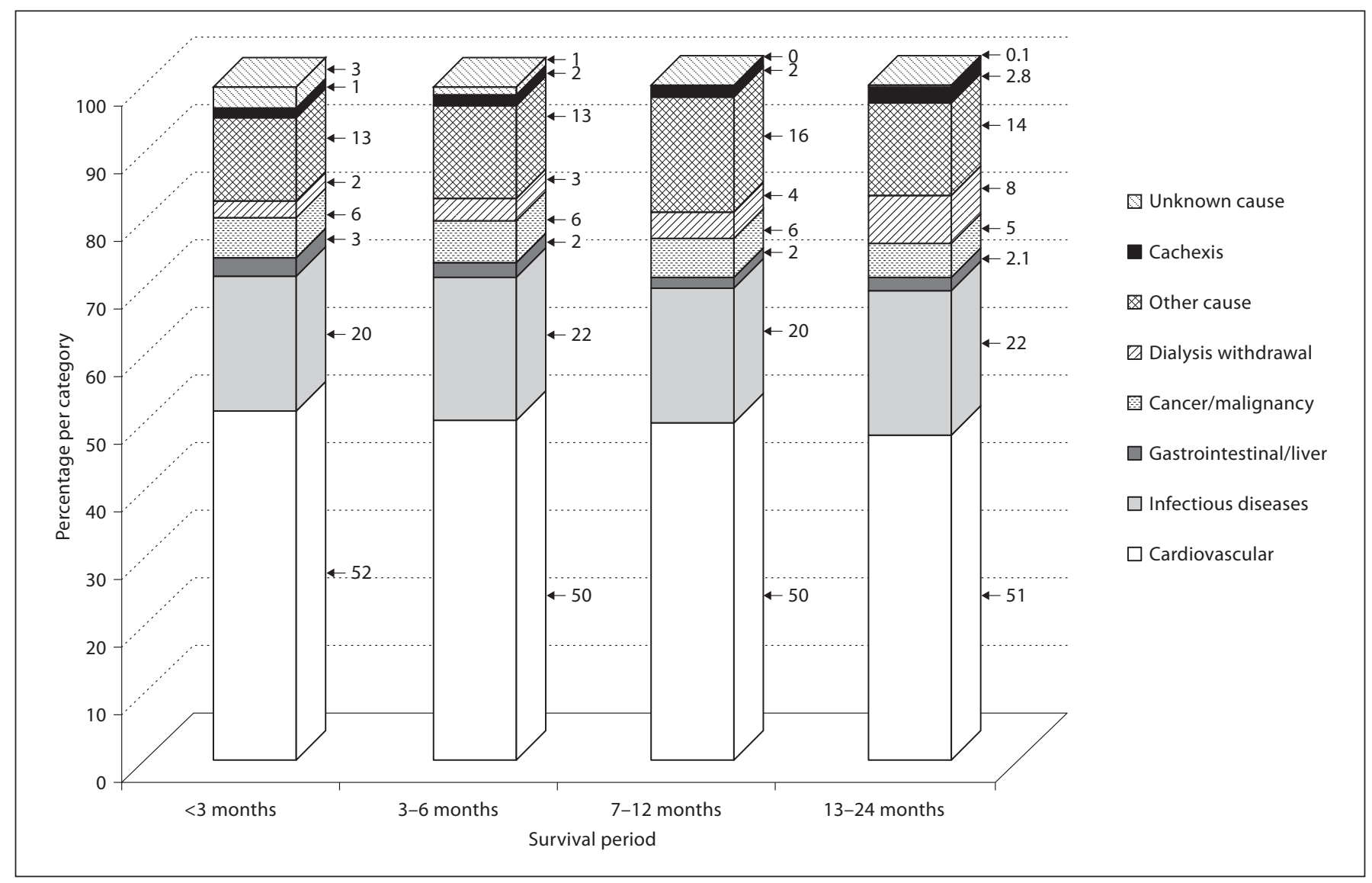

Fig. 3. Causes of death by the mortality periods for incident hemodialysis patients from the 5-year DaVita cohort, excluding patients with missing causes of death; $n=6,867$ (2,236 cases with no documented cause of death were excluded).

blacks and $12 \%$ Hispanics in contrast to $47 \%$ whites, $27 \%$ blacks, and 15\% Hispanics among survivors over 2 years (table 1), and was consistent with the survival advantage of minorities [21]. However, after case-mix adjustment, the advantage for African Americans during early dialysis period disappeared. Similar to the all-cause mortality result African Americans exhibited essentially the same pattern with cardiovascular mortality with practically no survival advantage over whites in early time periods, although a somewhat better survival in infection-related morality. The observation that African Americans had somewhat lower CVC use than whites (data not shown) might explain the higher infection-related mortality in whites. Hispanics, too, showed better survival in infectious deaths analyses; however, although whites had somewhat higher percentage of CHF history, Hispanics contributed to a higher percentage of patients with low serum albumin levels and CVC use similar to whites.
Both hypoalbuminemia and CVC access are known to be associated with infectious events and death. It might be that, while Hispanics were more likely to develop infections, they might have been less likely to die from them. Another explanation as to why Hispanics and, to some extent, African Americans had higher rates of hypoalbuminemia but lower infection-related mortality could be that, for most of the minorities, low albumin levels may be more indicative of malnutrition than infection [22].

An interesting finding was the seemingly paradoxical association between diabetes and lower mortality in the first months, which was previously reported by Bradbury et al. [3]. The possible explanation may be that they were more likely to see a physician on regular basis compared to nondiabetics and therefore may have been better prepared for the transitional period of early dialysis therapy. However, this survival advantage mitigated and even reversed over time probably because nondiabetics who sur- 
Table 2. Case-mix-adjusted death hazard ratios (95\% confidence intervals) for the association between mortality at different time periods and individual predictors for incident hemodialysis patients in 18,707 incident hemodialysis patients

\begin{tabular}{|c|c|c|c|c|}
\hline Age (decades) & $1.50(1.43-1.56)$ & $1.44(1.35-1.54)$ & $1.32(1.26-1.38)$ & $1.33(1.28-1.39)$ \\
\hline Gender (female vs. male) & $1.00(0.90-1.11)$ & $0.95(0.83-1.09)$ & $0.89(0.79-1.01)$ & $0.95(0.85-1.05)$ \\
\hline \multicolumn{5}{|l|}{ Race } \\
\hline Whites versus blacks & $1.12(0.97-1.28)$ & $1.01(0.86-1.18)$ & $1.33(1.16-1.52)$ & $1.29(1.14-1.46)$ \\
\hline Whites versus Hispanics & $1.09(0.94-1.27)$ & $1.24(1.02-1.52)$ & $1.53(1.23-1.85)$ & $1.33(1.14-1.56)$ \\
\hline \multicolumn{5}{|l|}{ Health insurance } \\
\hline Medicaid versus Medicare & $1.48(1.17-1.87)$ & $1.74(1.32-2.29)$ & $1.68(1.30-2.18)$ & $1.07(0.84-1.37)$ \\
\hline Other versus Medicare & $1.10(0.93-1.31)$ & $0.90(0.77-1.05)$ & $0.78(0.66-0.91)$ & $0.78(0.69-0.89)$ \\
\hline \multicolumn{5}{|l|}{ Marital status } \\
\hline Divorced versus married & $0.90(0.60-1.33)$ & $1.09(0.67-1.80)$ & $1.15(0.69-1.92)$ & $0.86(0.60-1.25)$ \\
\hline Single versus married & $1.39(1.16-1.65)$ & $1.33(1.10-1.60)$ & $1.19(0.94-1.51)$ & $1.08(0.88-1.34)$ \\
\hline Widowed versus married & $1.12(0.87-1.45)$ & $1.25(0.83-1.88)$ & $1.46(1.08-1.97)$ & $1.00(0.82-1.21)$ \\
\hline BMI (2 index points) & $0.92(0.90-0.94)$ & $0.93(0.91-0.95)$ & $0.94(0.92-0.96)$ & $0.92(0.91-0.94)$ \\
\hline \multicolumn{5}{|l|}{ Comorbidities } \\
\hline \multicolumn{5}{|l|}{ Cardiac disorders } \\
\hline Atherosclerotic heart disease & $1.09(0.97-1.23)$ & $0.96(0.83-1.11)$ & $1.04(0.92-1.18)$ & $1.05(0.93-1.18)$ \\
\hline Heart failure & $1.31(1.11-1.54)$ & $1.36(1.20-1.55)$ & $1.29(1.15-1.45)$ & $1.33(1.19-1.48)$ \\
\hline Other cardiac disease & $1.23(1.05-1.44)$ & $1.45(1.20-1.76)$ & $1.02(0.83-1.25)$ & $1.00(0.82-1.22)$ \\
\hline \multicolumn{5}{|l|}{ Vascular/pulmonary } \\
\hline Cerebrovascular disease & $1.07(0.92-1.24)$ & $1.09(0.91-1.31)$ & $1.08(0.91-1.28)$ & $1.27(1.09-1.48)$ \\
\hline Peripheral vascular disease & $1.11(0.98-1.27)$ & $1.27(1.08-1.49)$ & $1.28(1.10-1.49)$ & $1.16(1.01-1.33)$ \\
\hline COPD & $1.43(1.23-1.67)$ & $1.02(0.82-1.27)$ & $1.14(0.94-1.37)$ & $1.13(0.95-1.36)$ \\
\hline \multicolumn{5}{|l|}{ Other comorbidities } \\
\hline Cancer & $1.31(1.11-1.54)$ & $1.36(1.11-1.67)$ & $1.30(1.06-1.60)$ & $1.15(0.95-1.40)$ \\
\hline Non-ambulatory & $2.01(1.70-2.38)$ & $1.73(1.38-2.18)$ & $1.46(1.15-1.84)$ & $1.66(1.33-2.09)$ \\
\hline Calcium, mg/dl* & $1.14(1.07-1.22)$ & $1.09(1.00-1.18)$ & $1.11(1.04-1.19)$ & $1.03(0.97-1.09)$ \\
\hline Phosphorus, mg/dl ${ }^{*}$ & $1.06(1.02-1.10)$ & $1.03(0.99-1.08)$ & $1.04(1.00-1.09)$ & $1.01(0.97-1.05)$ \\
\hline nPCR (0.2 g/kg/day) & $1.08(1.03-1.13)$ & $0.94(0.89-1.00)$ & $0.93(0.89-0.97)$ & $0.96(0.92-1.00)$ \\
\hline $\mathrm{nPCR}>1.0 \mathrm{~g} / \mathrm{kg} /$ day & $1.21(1.06-1.38)$ & $0.96(0.8-1.14)$ & $0.89(0.74-1.07)$ & $0.99(0.87-1.13)$ \\
\hline $\operatorname{WBC}(5,000 / \mathrm{HPF} \uparrow)$ & $1.52(1.45-1.59)$ & $1.26(1.15-1.39)$ & $1.26(1.16-1.37)$ & $1.14(1.04-1.25)$ \\
\hline Lymphocytes $(10 \%)^{*}$ & $1.87(1.70-2.05)$ & $1.42(1.31-1.55)$ & $1.33(1.24-1.44)$ & $1.17(1.09-1.26)$ \\
\hline TIBC $(50 \mathrm{~g} / \mathrm{dl})^{*}$ & $1.51(1.43-1.59)$ & $1.40(1.31-1.49)$ & $1.30(1.22-1.38)$ & $1.20(1.14-1.26)$ \\
\hline Ferritin $(500 \mathrm{ng} / \mathrm{dl})^{*}$ & $1.24(1.20-1.28)$ & $1.23(1.18-1.29)$ & $1.27(1.21-1.33)$ & $1.20(1.14-1.28)$ \\
\hline Parathyroid hormone $(100 \mathrm{pg} / \mathrm{ml})$ & $0.96(0.94-0.97)$ & $0.97(0.95-0.99)$ & $0.99(0.97-1.01)$ & $0.99(0.97-1.01)$ \\
\hline ISAT $(10 \%)$ & $1.09(1.05-1.13)$ & $1.05(0.99-1.11)$ & $1.02(0.98-1.08)$ & $1.07(1.02-1.13)$ \\
\hline eGFR (each 1 increment) & $1.03(1.02-1.04)$ & $1.01(1.00-1.03)$ & $1.01(1.00-1.03)$ & $1.03(1.02-1.04)$ \\
\hline $\mathrm{eGFR}>10$ & $1.29(1.17-1.42)$ & $1.20(1.07-1.35)$ & $1.04(0.94-1.16)$ & $1.11(0.97-1.28)$ \\
\hline eGFR >15 & $1.43(1.27-1.60)$ & $1.09(0.93-1.28)$ & $1.29(1.12-1.48)$ & $1.02(0.83-1.24)$ \\
\hline
\end{tabular}

Note that deaths and person-times before and/or after each period are censored.

$\mathrm{AVF}=$ Arteriovenous fistula; graft $=$ arteriovenous graft COPD = chronic obstructive pulmonary disease; eGFR = estimated glomerular filtration rate .

* Decrease per unit change of serum level of laboratory parameter. 
Table 3. Case-mix-adjusted death hazard ratios (95\% confidence intervals) for the association between cardiovascular and infectionrelated mortality at different time periods and several individual predictors for incident hemodialysis patients in 18,707 incident hemodialysis patients (outcome 1: cardiovascular mortality, outcome 2: Infectious mortality)

\begin{tabular}{|c|c|c|c|c|}
\hline Predictor & Deaths $0-3$ months & Deaths $4-6$ months & Deaths 7-12 months & Deaths $13-24$ months \\
\hline \multicolumn{5}{|l|}{ Whites versus blacks } \\
\hline Cardiovascular mortality & $1.14(0.92-1.41)$ & $1.02(0.81-1.30)$ & $1.20(0.94-1.54)$ & $1.46(1.18-1.80)$ \\
\hline \multicolumn{5}{|l|}{ Whites versus Hispanics } \\
\hline Cardiovascular mortality & $1.28(0.98-1.66)$ & $1.16(0.84-1.62)$ & $1.75(1.28-2.39)$ & $1.60(1.22-2.10)$ \\
\hline Infectious mortality & $0.91(0.60-1.37)$ & $1.38(0.42-2.33)$ & $3.43(1.76-6.69)$ & $1.58(0.96-2.62)$ \\
\hline \multicolumn{5}{|l|}{ Heart failure } \\
\hline Cardiovascular mortality & $1.68(1.41-1.99)$ & $1.74(1.42-2.14)$ & $1.86(1.53-2.24)$ & $1.43(1.20-1.71)$ \\
\hline Infectious mortality & $1.56(1.14-2.14)$ & $1.76(1.24-2.50)$ & $1.17(0.80-1.70)$ & $1.69(1.24-2.31)$ \\
\hline \multicolumn{5}{|l|}{ CVC versus AVF + graft } \\
\hline Cardiovascular mortality & $2.45(1.74-3.44)$ & $2.09(1.57-2.80)$ & $1.58(1.31-1.93)$ & $1.59(1.30-1.97)$ \\
\hline Infectious mortality & $3.32(2.14-5.16)$ & $4.03(2.66-6.09)$ & $2.23(1.50-3.32)$ & $1.74(1.23-2.47)$ \\
\hline \multicolumn{5}{|l|}{ Albumin $<3.5$ versus $>3.5 \mathrm{~g} / \mathrm{dl}$} \\
\hline
\end{tabular}

Note that deaths and person-times before and/or after each period are censored.

$\mathrm{AVF}=$ Arteriovenous fistula; graft = arteriovenous graft.

vived the transitional period might be healthier than patients with diabetes.

Among clinical predictors, type of the vascular access was exceptionally strongly associated with mortality across all studied periods but in particular during the first 90 days, including both among cardiovascular and infection-related deaths. Although our finding was consistent with previous reports indicating that CVC was associated with higher mortality in hemodialysis patients [9-12], our study uniquely shows that the association of vascular access type with mortality is the strongest at the time of dialysis initiation and decreased over time, which suggests the importance of replacing CVC with a more permanent access within the first few weeks - if not days - of dialysis therapy. Our results are consistent with findings from the Dialysis Outcomes and Practice Patterns Study (DOPPS) reporting higher mortality for patients with CVC access comparing to other types. That study found that US patients had 36 to $43 \%$ higher mortality risk due to widespread use of CVC use compared to European countries and about $30 \%$ higher when compared to Japan [23].

The resilient role of low serum albumin level in predicting high mortality of dialysis patients has been previously identified $[3,24,25]$; this is verified in our current study. A 10-year cohort study from Japan reported that patients with serum albumin levels $>3.8 \mathrm{~g} / \mathrm{dl}$ consistently had better survival [24]. We found $21 \%$ increase in mortality in the first 90 days per $0.2 \mathrm{~g} / \mathrm{d}$ l lower serum albumin compared to $12 \%$ during the $12-24$-month period. We found that infection-related and cardiovascular mortality rates were, respectively, 5-fold and 2 -fold higher among patients with low serum albumin levels $<3.5 \mathrm{~g} / \mathrm{dl}$ compared to higher levels. We also found that, among patients with no history of CHF and hypoalbuminemia, the percentage of deaths related to infections was the highest especially during the first 6 months on dialysis (19-21\%), while patients with history of CHF and serum albumin $>3.5 \mathrm{~g} / \mathrm{dl}$ accounted for only $8 \%$ of infectionrelated deaths in the first 3 months of dialysis treatment (table 3). Although hypoalbuminemia was strongly associated with both cardiovascular and infection-related mortality, it seems that, among patients who died from cardiovascular disease, CHF, which is usually due to volume overload, could be responsible for the patient outcomes especially during the early months of the dialysis treatment. Patients with CHF had higher cardiovascular mortality than patients without it, and the highest cardiovascular mortality of $76 \%$ was observed during the first 3 months of dialysis treatment in patients with CHF and serum albumin $>3.5 \mathrm{~g} / \mathrm{dl}$. 
In a recent study from the UK Renal National Registry cohort, using several models for predicting the first 3 -year survival of incident dialysis patients, older age, white race, diabetes mellitus and other primary causes of ESRD, history of cardiovascular disease and smoking were predictive of increased mortality [27]. Among laboratory parameters, serum levels of albumin, hemoglobin, and calcium were also predictors of 2-year mortality [27]. These findings are somewhat similar to our results. We also found an elevated mortality risk of $58 \%$ for patients with blood hemoglobin $<10 \mathrm{~g} / \mathrm{ml}$ during the first 3 months of dialysis treatment.

Our study should be qualified for including only DaVita patients rather than the entire national dialysis population. However, DaVita patients are likely good representatives of average ESRD patients. We purposely excluded the incident patients who initiated their first week of therapy elsewhere to mitigate selection bias. It can be argued that there could be differences between the patients who started dialysis at an inpatient versus outpatient facility. Nevertheless, the annualized mortality in our cohort for the first 3 months was about $30 \%$, which was consistent with mortality reported for US patients in the same period [2]. We had no reliable information about patient visiting nephrologists prior to dialysis initiation ( $80 \%$ missing values), which precluded examination of this potential predictor of early mortality.

\section{Conclusion}

Incident hemodialysis patients have the highest mortality during the first 6 months of dialysis therapy, in particular in the first 2 months, and cardiovascular disease is the most common cause of death. Use of CVC as vascular access and hypoalbuminemia $<3.5 \mathrm{mg} / \mathrm{dl}$ each explains one third of all deaths in the first 90 days. Hence, replacing or avoiding CVC and improving hypolabuminemia could theoretically reduce early dialysis death by $30 \%$. These findings warrant imminent design of clinical trials to examine interventions to target imminent arteriovenous fistula placement and to increase serum albu$\mathrm{min}$ in order to reduce early death among incident dialysis patients. Low hemoglobin level could explain 9\% of deaths in the first 3 months indicating the potential role of proper anemia management in improving outcomes of new hemodialysis patients.

\section{Acknowledgement}

We thank DaVita Clinical Research ${ }^{\circledR}$ (DCR) for providing the clinical data, analysis and review for this research project and for advancing the knowledge and practice of kidney care.

\section{Funding Source}

The study was supported by a research grant from the National Institute of Diabetes, Digestive and Kidney Disease of the National Institutes of Health R01 DK078106, a philanthropist grant from Mr. Harold Simmons and a research grant from DaVita Clinical Research. O.A.A. was supported by a Veni career grant No. 916.96.059 from the Netherlands Organization for Scientific Research (NWO).

\section{Disclosure Statement}

The authors declare that there are no potential conflicts of interest.

\section{References}

1 US Renal Data System: USRDS 2009 Annual Data Report: Atlas of Chronic Kidney Disease and End-Stage Renal Disease in the United States National Institute of Health Volume 1. 2009, National Institute of Health, National Institute of Diabetes and Digestive and Kidney Disease: Bethesda, MD.

2 Moretti HD, Johnson AM, Keeling-Hathaway TJ: Effects of protein supplementation in chronic hemodialysis and peritoneal dialysis patients. J Ren Nutr 2009;19:298-303.

\footnotetext{
-3 Bradbury BD, et al: Predictors of early mortality among incident US hemodialysis patients in the Dialysis Outcomes and Practice Patterns Study (DOPPS). Clin J Am Soc Nephrol 2007;2:89-99.

4 Evans M, et al: No survival benefit from early-start dialysis in a population-based, inception cohort study of Swedish patients with chronic kidney disease. J Intern Med 2011;269:289-298.

$\checkmark 5$ Lassalle M, et al: Age and comorbidity may explain the paradoxical association of an early dialysis start with poor survival. Kidney Int 2010;77:700-707.
}

6 Molnar MZ, et al: Estimated glomerular filtration rate at re-initiation of dialysis and mortality in failed kidney transplant recipients. Nephrol Dial Transplant 2012 [in press].

7 Cooper BA, et al: A randomized, controlled trial of early versus late initiation of dialysis. N Engl J Med 2010;363:609-619.

8 Molnar MZ, et al: Early versus late dialysis initiation in transplant-naïve and failed kidney transplant patients. Nat Rev Nephrol 2012 [in press].

9 Wasse H: Catheter-related mortality among ESRD patients. Semin Dial 2008;21:547-549. 
10 Wasse H, et al: Predictors of central venous catheter use at the initiation of hemodialysis. Semin Dial 2008;21:346-351.

11 Gallieni M, Martini A, Mezzina N: Dialysis access: an increasingly important clinical issue. Int J Artif Organs 2009;32:851-856.

$\checkmark 12$ Collins AJ, et al: The state of chronic kidney disease, ESRD, and morbidity and mortality in the first year of dialysis. Clin J Am Soc Nephrol 2009;4(suppl 1):S5-S11.

13 Researcher's Guide to the USRDS Database - 2008 ADR Edition. 2008, United States Renal Data System National Institutes of Health; National Institute of Diabetes and Digestive Disease and Kidney Disease Division of Kidney Urologic and Hematologic Diseases: Bethesda, MD

14 Miller JE, et al: Association of hemodialysis treatment time and dose with mortality and the role of race and sex. Am J Kidney Dis 2010;55:100-112.

15 Shinaberger CS, et al: Longitudinal associations between dietary protein intake and survival in hemodialysis patients. Am J Kidney Dis 2006;48:37-49.

16 Kalantar-Zadeh K, et al: Malnutrition-inflammation complex syndrome in dialysis patients: causes and consequences. Am J Kidney Dis 2003;42:864-881.
17 Roalfe AK, Holder RL, Wilson S: Standardisation of rates using logistic regression: a comparison with the direct method. BMC Health Serv Res 2008;8:275.

18 Rothman KJ, Greenland S, Lash TL: Modern Epidemiology. 3rd ed. 2008, Philladelphia, PA: Wolters Kluwer Lippincott Williams \& Wilkins.

19 Greenland S: Re: 'Confidence limits made easy: interval estimation using a substitution method'. Am J Epidemiol 1999;149:884; author reply 885-886.

20 Plantinga LC, et al: Early, intermediate, and long-term risk factors for mortality in incident dialysis patients: the Choices for Healthy Outcomes in Caring for ESRD (CHOICE) Study. Am J Kidney Dis 2007;49: 831-840.

21 Kalantar-Zadeh K, et al: Impact of race on hyperparathyroidism, mineral disarrays, administered vitamin D mimetic, and survival in hemodialysis patients. J Bone Miner Res 2010;25:2724-2734.

22 Ricks J, Streja L, Greenland S: Race, age, and mortality among patients undergoing dialysis. JAMA 2011;306:2215; author reply 22152216.
23 Pisoni RL, et al: Facility hemodialysis vascular access use and mortality in countries participating in DOPPS: an instrumental variable analysis. Am J Kidney Dis 2009;53:475491.

24 Kato A, et al: Comparison of serum albumin, C-reactive protein and carotid atherosclerosis as predictors of 10-year mortality in hemodialysis patients. Hemodial Int 2010;14: 226-232.

25 Capelli JP, Kushner H: Correlates affecting survival in chronic hemodialysis patients: the combined impact of albumin and high hemoglobin levels on improving outcomes, local and national results. Hemodial Int 2008;12:450-462.

26 Bross R, et al: Association of serum total iron-binding capacity and its changes over time with nutritional and clinical outcomes in hemodialysis patients. Am J Nephrol 2009;29:571-581.

27 Wagner M, et al: Predicting mortality in incident dialysis patients: an analysis of the United Kingdom renal registry. Am J Kidney Dis 2011;57:894-902. 